Annals of Pure and Applied Mathematics

Vol. 16, No. 2, 2018, 345-352

ISSN: 2279-087X (P), 2279-0888(online)

Published on 22 February 2018

www.researchmathsci.org

DOI: http://dx.doi.org/10.22457/apam.v16n2a11

Annals of

Pure and Applied

Mathematics

\title{
Some New Uniqueness Results of Solutions to Nonlinear Fractional Integro-Differential Equations
}

\author{
Mohammed S. Abdo ${ }^{1}$ and Satish K. Panchal ${ }^{2}$
}

Department of Mathematics

Dr. Babasaheb Ambedkar Marathwada University

Aurangabad (M.S.), 431004 India.

1E-mail: msabdo1977@ gmail.com; ${ }^{2}$ E-mail: drpanchalsk@ gmail.com

Received 9 February 2018; accepted 22 February 2018

Abstract. The aim of this paper is to prove the new uniqueness of solutions for nonlinear integr-odifferential equations with fractional of arbitrary order which includes the Caputo fractional operator derivative. The techniques employed are a variety of tools such as fractional calculus and its properties, Bihari's inequality, and Banach contraction mapping principle.

Keywords: Fractional derivative and integral, Integro-differential equations, Integral inequalities, fixed point theorem.

AMS Mathematics Subject Classification (2010): 26A33, 26D10, 47H10

\section{Introduction}

This paper is concerned with the uniqueness results for fractional integro differential equations of the type

$$
{ }^{c} D_{0^{+}}^{\alpha} y(t)=a(t) y(t)+f(t, y(t))+\int_{0}^{t} K(t, s, y(s)) d s, t \in[0, b],
$$

with initial condition

$$
y(0)=y_{0},
$$

where $0<\alpha \leq 1, a \in C([0, b], \mathbb{R}), f \in C([0, b] \times \mathbb{R}, \mathbb{R})$.

$K \in C([0, b] \times[0, b] \times \mathbb{R}, \mathbb{R})$ and satisfying some conditions which will be mentioned later and ${ }^{c} D^{\alpha}$ is the Caputo fractional derivative. The study of fractional differential equations is related to the extensive applications of fractional calculus in mechanics, physics, chemical technology, biotechnology, and so forth.

In latest years, there has been an interest in the look at of the theory fractional differential equations, it has seen significant improvement, see as an instance the monographs of Delboso and Rodino [8], Diethelm [9], Kilbas et al. [10], Miller and Ross [11] Oldham and Spanier [13] and the references therein. Recently, uniqueness standards for the various fractional integro-differential equations have been taken into consideration by way some authors, for greater info, (see $[1,2,3,4,5,6,7,12,16]$ ). For example in [12], 


\section{S. Abdo and S. K. Panchal}

Momani et al. studied the local uniqueness result by applying Bihari's inequality for the following problem

$$
\begin{gathered}
{ }^{c} D^{\alpha} y(t)=f(t, y(t))+\int_{t_{0}}^{t} K(t, s, y(s)) d s, \\
y(0)=y_{0},
\end{gathered}
$$

where $0<\alpha \leq 1, f \in C\left([0, b] \times \mathbb{R}^{n}, \mathbb{R}^{n}\right), \quad K \in C\left([0, b] \times[0, b] \times \mathbb{R}^{n}, \mathbb{R}^{n}\right)$ and ${ }^{c} D^{\alpha}$ is the Caputo fractional operator. In [6], Balachandran and Trujillo, discussed the existence and uniqueness of solutions of quasilinear and semilinear of integro-differential equations with fractional order in Banach space by means of Banach fixed point theorem. In this paper, we prove the uniqueness results of the fractional integro-differential equations (1.1)-(1.2) with the aid of using Bihari's inequality and Banach contraction principle.

The rest of this paper is split as follows. In Section 2, we present some mathematical tools and listing the assumptions which have advantage on this paper. Section 3 is dedicated to proving the uniqueness of solutions for problem (1.1)-(1.2). Finally, the conclusion and future work are given in Section 4.

\section{Some mathematical tools}

In this section, some required notations, definitions and a lemmas are given.

Let $J=[0, b]$ and $C(J, \mathbb{R})$ be the Banach space endowed with the infinity norm $\|g\|_{\infty}=\sup \{|g(t)|: t \in J\}$, for any $g \in C(J, \mathbb{R})$, we also $C^{n}\left(J, \mathbb{R}^{+}\right)$be space of all real valued continuous function which are continuously differentiable on $J$.

Definition 2.1. [10] The Riemann-Liouville fractional integral of order $\alpha>0$ of the function $g \in C(J, \mathbb{R})$ is given by

$$
I_{0^{+}}^{\alpha} g(t)=\frac{1}{\Gamma(\alpha)} \int_{0}^{t}(t-s)^{\alpha-1} g(s) d s, \quad t \in J,
$$

where $\Gamma$ denotes the Gamma function.

Definition 2.2. [10] Let $n-1<\alpha<n$. The Caputo's fractional operator of the function $g \in C^{n}(J, \mathbb{R})$ is defined as

$$
{ }^{c} D_{0^{+}}^{\alpha} g(t)=\frac{1}{\Gamma(n-\alpha)} \int_{0}^{t}(t-s)^{n-\alpha-1} \frac{d^{n}}{d t^{n}} g(s) d s=I_{0^{+}}^{n-\alpha-1} \frac{d^{n}}{d t^{n}} g(t), \quad t \in J .
$$

In particular, if $0<\alpha<1$, then ${ }^{c} D_{0^{+}}^{\alpha} g(t)=I_{0^{+}}^{1-\alpha} \frac{d}{d t} g(t), t \in J$.

Lemma 2.1. [10,15] For $\alpha, \beta>0$ and $g, h$ are appropriate functions then, for $t \in J$, we have,

$$
\begin{aligned}
& \text { 1) }{ }^{c} D_{0^{+}}^{\alpha} I_{0^{+}}^{\alpha} g(t)=g(t) . \\
& \text { 2) } I_{0^{+}}^{\alpha c} D_{0^{+}}^{\alpha} g(t)=g(t)-g(0), \quad 0<\alpha<1 .
\end{aligned}
$$


Some New Uniqueness Results of Solutions to Nonlinear Fractional Integro-Differential Equations

3) $I_{0^{+}}^{\alpha} I_{0^{+}}^{\beta} g(t)=I_{0^{+}}^{\alpha+\beta} g(t)=I_{0^{+}}^{\beta} I_{0^{+}}^{\alpha} g(t)$.

4) $I_{0^{+}}^{\alpha}(g(t)+h(t))=I_{0^{+}}^{\alpha} g(t)+I_{0^{+}}^{\alpha} h(t)$.

5) ${ }^{c} D_{0^{+}}^{\alpha} C=0$, where $C$ is a constant.

Lemma 2.2. [17] (Banach contraction principle). Let $K$ be a non-empty closed subset of a Banach space $X$, then each contraction mapping $T: K \rightarrow K$ has a unique fixed point.

Lemma 2.3. [14] (Bihari's inequality). Let $g:[a, b] \rightarrow \mathbb{R}^{+}$be a continuous function that satisfies the inequality

$$
g(t) \leq A+\int_{a}^{t} K(s) \psi(g(s)) d s, t \in[a, b],
$$

where $A \geq 0, \quad K:[a, b] \rightarrow \mathbb{R}^{+}$is continuous and $\psi:[0,+\infty) \rightarrow(0,+\infty)$ is continuous and monotone-increasing. Then the following inequality hold

$$
g(t) \leq \Psi^{-1}\left[\Psi(A)+\int_{a}^{t} K(s) d s\right], t \in[a, b],
$$

where $\Psi: \mathbb{R} \rightarrow \mathbb{R}$ is given by $\Psi(x)=\int_{x_{0}}^{x} \frac{d s}{\psi(s)}, \quad x \in \mathbb{R}$.

\section{Main results}

In this section, we shall exhibit and demonstrator the uniqueness results of problem (1.1)(1.2). Before beginning and proving our main results, we present the following lemma:

Lemma 3.1. The initial value problem (1.1)-(1.2) is equivalent to the nonlinear integral equation

$$
\begin{aligned}
y(t)= & y_{0}+\frac{1}{\Gamma(\alpha)} \int_{0}^{t}(t-s)^{\alpha-1} a(s) y(s) d s+\frac{1}{\Gamma(\alpha)} \int_{0}^{t}(t-s)^{\alpha-1} f(s, y(s)) d s \\
& +\frac{1}{\Gamma(\alpha)} \int_{0}^{t}(t-s)^{\alpha-1} \int_{s}^{t} K(\tau, s, y(s)) d \tau d s, \quad t \in J .
\end{aligned}
$$

In other hand, each solution of the integral equation (3.1) is likewise a solution of initial value problem (1.1)-(1.2) and vice versa.

Proof: It can be proved effortlessly by effect the integral operator $I_{0^{+}}^{\alpha}$ described in the Definition 2.1 to both sides of Eq.(1.1), so according of Lemma 2.1 and switch the order of integration (Fubini's theorem) to get the integral equation (3.1).

Let us list the hypotheses:

(A1) $a: J \rightarrow \mathbb{R}$ is continuous.

(A2) $f: J \times \mathbb{R} \rightarrow \mathbb{R}$ is continuous such that

$$
|f(t, u)-f(t, v)| \leq \psi(|u-v|), t \in J, u, v \in \mathbb{R} .
$$

(A3) $K: J \times J \times \mathbb{R} \rightarrow \mathbb{R}$, is continuous on $D$ such that 
M. S. Abdo and S. K. Panchal

$$
\int_{s}^{t}\left|K(\tau, s, u(s))-K_{1}(\tau, s, v(s))\right| d \tau \leq M \psi(|u-v|),
$$

for $t \in J, u, v \in \mathbb{R}$, where $\psi: \mathbb{R}^{+} \rightarrow \mathbb{R}^{+}$is nondecreasing continuous function with $\psi(0)=0$ and $\int_{0}^{R} \frac{d x}{\psi(x)}=+\infty, 0<x<R, D=\{(t, s): 0 \leq s \leq t \leq b\}$ and $M$ is a positive constant.

Our first result depends on Bihari's inequality.

Theorem 3.1. Assume that the hypotheses (A1),(A2) and (A3) hold. If $0 \leq \frac{\|a\|_{\infty} b^{\alpha}}{\Gamma(\alpha+1)}<1$, then the fractional integro-differential equation (1.1)-(1.2) has a unique solution in $C(J, \mathbb{R})$.

Proof: By Lemma 3.1, we know that the function $y$ is a solution to (1.1)-(1.2) iff $y$ satisfies

$$
\begin{aligned}
y(t)= & y_{0}+\frac{1}{\Gamma(\alpha)} \int_{0}^{t}(t-s)^{\alpha-1} a(s) y(s) d s+\frac{1}{\Gamma(\alpha)} \int_{0}^{t}(t-s)^{\alpha-1} f(s, y(s)) d s \\
& +\frac{1}{\Gamma(\alpha)} \int_{0}^{t}(t-s)^{\alpha-1} \int_{s}^{t} K(\tau, s, y(s)) d \tau d s, \quad t \in J .
\end{aligned}
$$

Let $y_{1}, y_{2} \in C(J, \mathbb{R})$ and for any $t \in J$ such that

$$
\begin{aligned}
y_{1}(t)= & y_{0}+\frac{1}{\Gamma(\alpha)} \int_{0}^{t}(t-s)^{\alpha-1} a(s) y_{1}(s) d s+\frac{1}{\Gamma(\alpha)} \int_{0}^{t}(t-s)^{\alpha-1} f\left(s, y_{1}(s)\right) d s \\
& +\frac{1}{\Gamma(\alpha)} \int_{0}^{t}(t-s)^{\alpha-1} \int_{s}^{t} K\left(\tau, s, y_{1}(s)\right) d \tau d s
\end{aligned}
$$

and

$$
\begin{aligned}
y_{2}(t)= & y_{0}+\frac{1}{\Gamma(\alpha)} \int_{0}^{t}(t-s)^{\alpha-1} a(s) y_{2}(s) d s+\frac{1}{\Gamma(\alpha)} \int_{0}^{t}(t-s)^{\alpha-1} f\left(s, y_{2}(s)\right) d s \\
& +\frac{1}{\Gamma(\alpha)} \int_{0}^{t}(t-s)^{\alpha-1} \int_{s}^{t} K\left(\tau, s, y_{2}(s)\right) d \tau d s .
\end{aligned}
$$

Consequently, by (A1), (A2) and (A3), then for $t \in J$, we have

$$
\begin{aligned}
\left|y_{1}(t)-y_{2}(t)\right| \leq & \frac{1}{\Gamma(\alpha)} \int_{0}^{t}(t-s)^{\alpha-1}|a(s)|\left|y_{1}(s)-y_{2}(s)\right| d s \\
& +\frac{1}{\Gamma(\alpha)} \int_{0}^{t}(t-s)^{\alpha-1}\left|f\left(s, y_{1}(s)\right)-f\left(s, y_{2}(s)\right)\right| d s \\
& +\frac{1}{\Gamma(\alpha)} \int_{0}^{t}(t-s)^{\alpha-1} \int_{s}^{t}\left|K\left(\tau, s, y_{1}(s)\right)-K_{1}\left(\tau, s, y_{2}(s)\right)\right| d \tau d s \\
& \leq \frac{1}{\Gamma(\alpha)} \int_{0}^{t}(t-s)^{\alpha-1} \sup _{s \in J}|a(s)|\left|y_{1}(s)-y_{2}(s)\right|
\end{aligned}
$$


Some New Uniqueness Results of Solutions to Nonlinear Fractional Integro-Differential Equations

$$
\begin{aligned}
& +\frac{1}{\Gamma(\alpha)} \int_{0}^{t}(t-s)^{\alpha-1} \psi\left(\left|y_{1}(s)-y_{2}(s)\right|\right) d s \\
& +\frac{1}{\Gamma(\alpha)} \int_{0}^{t}(t-s)^{\alpha-1} M \psi\left(\left|y_{1}(s)-y_{2}(s)\right|\right) d s \\
& \leq \frac{\|a\|_{\infty} b^{\alpha}}{\Gamma(\alpha+1)}\left|y_{1}(t)-y_{2}(t)\right|+\frac{1+M}{\Gamma(\alpha)} \int_{0}^{t}(t-s)^{\alpha-1} \psi\left(\left|y_{1}(s)-y_{2}(s)\right|\right) d s \\
& \leq \frac{1+M}{1-\frac{\|a\|_{\infty} b^{\alpha}}{\Gamma(\alpha+1)}} \frac{1}{\Gamma(\alpha)} \int_{0}^{t}(t-s)^{\alpha-1} \psi\left(\left|y_{1}(s)-y_{2}(s)\right|\right) d s \\
& <\varepsilon+\frac{M}{1-\frac{\|a\|_{\infty} b^{\alpha}}{\Gamma(\alpha+1)}} \frac{1}{\Gamma(\alpha)} \int_{0}^{t}(t-s)^{\alpha-1} \psi\left(\left|y_{1}(s)-y_{2}(s)\right|\right) d s,
\end{aligned}
$$

where $\varepsilon=\frac{1}{1-\frac{|l| \|_{0} \omega^{\alpha}}{\Gamma(\alpha+1)}}>0$. We can now apply Lemma 2.3 to obtain

$$
\begin{aligned}
\left|y_{1}(t)-y_{2}(t)\right| & \leq \Psi^{-1}\left[\Psi(\varepsilon)+\frac{M}{1-\frac{\|a\|_{b^{\alpha}}}{\Gamma(\alpha+1)}} \frac{1}{\Gamma(\alpha)} \int_{0}^{t}(t-s)^{\alpha-1} d s\right] \\
& \leq \Psi^{-1}\left[\Psi(\mathcal{E})+\frac{M}{1-\frac{\|a\|_{0} b^{\alpha}}{\Gamma(\alpha+1)}} \frac{b^{\alpha}}{\Gamma(\alpha+1)}\right],
\end{aligned}
$$

where $\Psi($.$) is a primitive of \frac{1}{\psi(.)}$, and $\Psi^{-1}($.$) is called the inverse of \Psi($.$) .$

It follows that $\Psi^{-1}\left[\Psi(\varepsilon)+\frac{1+M}{1-|| a \|_{\infty}} \frac{b^{\alpha}}{\Gamma(\alpha+1)}\right] \rightarrow 0$. (see [12]). Consequently, $y_{1}(t)=y_{2}(t)$, for $t \in[0, b]$. So, $y(t) \in C(J, \mathbb{R})$ is the unique solution to fractional integro differential equation (1.1)-(1.2) and the proof is completed.

Our second result depend on the Banach contraction principle. For sake of convenient, we introduce the following assumptions

(B1) $a: J \rightarrow \mathbb{R}$ is continuous.

(B2) $f: J \times \mathbb{R} \rightarrow \mathbb{R}$ is continuous and there exists a constant $L>0$ such that

$$
|f(t, u)-f(t, v)| \leq L\|u-v\|, t \in J, u, v \in \mathbb{R} .
$$

(B3) $K: J \times J \times \mathbb{R} \rightarrow \mathbb{R}$ is continuous and there exists a constant $L^{*}>0$ such that

$$
\left|K(\tau, s, u(s))-K_{1}(\tau, s, v(s))\right| \leq L^{*}\|u-v\|,(t, s) \in D, u, v \in \mathbb{R},
$$

where $D$ is defined as in Theorem 3.1 . 
Theorem 3.2. Assume that the hypotheses (B1)-(B3) are satisfied. And let $\zeta$ and $\eta$ be two positive real numbers such that $0<\zeta<1$ and

$$
\begin{gathered}
\left(\frac{\|a\|_{\infty}+L}{\Gamma(\alpha+1)}+\frac{L^{*} b}{(\alpha+1) \Gamma(\alpha)}\right) b^{\alpha}=\zeta, \\
\left|y_{0}\right|+\left(\frac{q}{\Gamma(\alpha+1)}+\frac{q^{*} b}{(\alpha+1) \Gamma(\alpha)}\right) b^{\alpha}=(1-\zeta) \eta .
\end{gathered}
$$

Then the fractional integro-differential equation (1.1)-(1.2) has a unique solution continuous on $[0, b]$, where $q=\max |f(t, 0)|: t \in J\}$ and $\left.q^{*}=\max |K(\tau, s, 0)|:(\tau, s) \in D\right\}$.

Proof: Let the operator $T: C(J, \mathbb{R}) \rightarrow C(J, \mathbb{R})$ be defined by

$$
\begin{aligned}
(T y)(t)= & y_{0}+\frac{1}{\Gamma(\alpha)} \int_{0}^{t}(t-s)^{\alpha-1} a(s) y(s) d s+\frac{1}{\Gamma(\alpha)} \int_{0}^{t}(t-s)^{\alpha-1} f(s, y(s)) d s \\
& +\frac{1}{\Gamma(\alpha)} \int_{0}^{t}(t-s)^{\alpha-1} \int_{s}^{t} K(\tau, s, y(s)) d \tau d s .
\end{aligned}
$$

and define $B_{\eta}=\left\{y \in C(J, \mathbb{R}):\|y\|_{\infty} \leq \eta\right\}$ for some $\eta>0$. Now, we need to prove that the operator $T$ has a fixed point on $B_{\eta} \subset C(J, \mathbb{R})$. This fixed point is the unique solution of (1.1)-(1.2). In order that, we present the proof in two steps:

Step 1: We show that $T B_{\eta} \subset B_{\eta}$.

By the hypotheses, then for any $y \in B_{\eta}$ and for $t \in J$, we have

$$
\begin{aligned}
|(T y)(t)| \leq & \left|y_{0}\right|+\frac{1}{\Gamma(\alpha)} \int_{0}^{t}(t-s)^{\alpha-1}|a(s)||y(s)| d s+\frac{1}{\Gamma(\alpha)} \int_{0}^{t}(t-s)^{\alpha-1}|f(s, y(s))| d s \\
& +\frac{1}{\Gamma(\alpha)} \int_{0}^{t}(t-s)^{\alpha-1} \int_{s}^{t}|K(\tau, s, y(s))| d \tau d s \\
\leq & \left|y_{0}\right|+\frac{1}{\Gamma(\alpha)} \int_{0}^{t}(t-s)^{\alpha-1}\|a\|_{\infty}\|y\|_{\infty} d s \\
& +\frac{1}{\Gamma(\alpha)} \int_{0}^{t}(t-s)^{\alpha-1}(|f(s, y(s))-f(s, 0)|+|f(s, 0)|) d s \\
& +\frac{1}{\Gamma(\alpha)} \int_{0}^{t}(t-s)^{\alpha-1} \int_{s}^{t}(|K(\tau, s, y(s))-K(\tau, s, 0)|+|K(\tau, s, 0)|) d \tau d s \\
& \leq\left|y_{0}\right|+\frac{b^{\alpha}\|a\|_{\infty} \eta}{\Gamma(\alpha+1)}+\frac{b^{\alpha}}{\Gamma(\alpha+1)}(L \eta+q)+\frac{b^{\alpha+1}}{(\alpha+1) \Gamma(\alpha)}\left(L^{*} \eta+q^{*}\right)
\end{aligned}
$$


Some New Uniqueness Results of Solutions to Nonlinear Fractional Integro-Differential Equations

$$
\begin{aligned}
& =\left|y_{0}\right|+\left(\frac{q}{\Gamma(\alpha+1)}+\frac{q^{*} b}{(\alpha+1) \Gamma(\alpha)}\right) b^{\alpha}+\left(\frac{\|a\|_{\infty}+L}{\Gamma(\alpha+1)}+\frac{L^{*} b}{(\alpha+1) \Gamma(\alpha)}\right) b^{\alpha} \eta \\
& =(1-\zeta) \eta+\zeta \eta=\eta .
\end{aligned}
$$

It follows that $\|T y\|_{\infty} \leq \eta$, this implise that $T y \in B_{\eta}$ which leads to $T B_{\eta} \subset B_{\eta}$.

Step 2: We shall show that $T: B_{\eta} \rightarrow B_{\eta}$ is a contraction mapping. Indeed, through the assumptions, then for any $y, y^{*} \in B_{\eta}$ and for $t \in J$, we can write

$$
\begin{aligned}
& \left|(T y)(t)-\left(T y^{*}\right)(t)\right| \\
\leq & \frac{1}{\Gamma(\alpha)} \int_{0}^{t}(t-s)^{\alpha-1}\left|a(s) \| y(s)-y^{*}(s)\right| d s+\frac{1}{\Gamma(\alpha)} \int_{0}^{t}(t-s)^{\alpha-1}\left|f(s, y(s))-f\left(s, y^{*}(s)\right)\right| d s \\
& +\frac{1}{\Gamma(\alpha)} \int_{0}^{t}(t-s)^{\alpha-1} \int_{s}^{t}\left|K(\tau, s, y(s))-K\left(\tau, s, y^{*}(s)\right)\right| d \tau d s \\
\leq & \frac{b^{\alpha}\|a\|_{\infty}}{\Gamma(\alpha+1)}\left\|y-y^{*}\right\|+\frac{b^{\alpha} L}{\Gamma(\alpha+1)}\left\|y-y^{*}\right\|+\frac{b^{\alpha+1} L^{*}}{(\alpha+1) \Gamma(\alpha)}\left\|y-y^{*}\right\| \\
= & \left(\frac{\|a\|_{\infty}+L}{\Gamma(\alpha+1)}+\frac{L^{*} b}{(\alpha+1) \Gamma(\alpha)}\right) b^{\alpha}\left\|y-y^{*}\right\| \\
= & \zeta\left\|y-y^{*}\right\| .
\end{aligned}
$$

Since $\zeta<1$, we get

$$
\left\|T y-T y^{*}\right\|_{\infty} \leq\left\|y-y^{*}\right\| .
$$

This implies that $T$ is contraction mapping. As consequence of Lemma 2.2, there exists a fixed point $y \in C(J, \mathbb{R})$ such that $T y=y$ which is the unique solution of (1.1)-(1.2) on $J$. This proves the required.

\section{Conclusion and future work}

The main purpose of this paper was to present new uniqueness results for nonlinear fractional integro-differential with respect to the Caputo fractional operator, highlighting the method which has been used by Momani et al. [12] to some results. The techniques used to prove our results are a variety of tools such as fractional calculus and its properties, Bihari's inequality and Banach fixed point theorem.

The possible generalization is to consider the problem (1.1)-(1.2) on Banach space and determine the conditions that be fit closer to obtain the best results. As another proposal, considering the Caputo type fractional derivative with respect to another function. These suggestions will be treated in the future. 
M. S. Abdo and S. K. Panchal

Acknowledgements. The authors are very grateful to referees for the valuable remarks and comments that improved this paper.

\section{REFERENCES}

1. M.S.Abdo and S.K.Panchal, Existence and continuous dependence for fractional neutral functional differential equations, J. Math. Model., 5 (2017) 153-170.

2. B.Ahmad and J.J.Nieto, Existence results for nonlinear boundary value problems of fractional integro-differential equations with integral boundary conditions, Boundary Value Problem, 2009 (2009) 708576.

3. B.Ahmad and S.Sivasundaram, Some existence results for fractional integrodifferential equations with nonlinear conditions, Communications Appl. Anal., 12 (2008) 107-112.

4. A.Anguraj, P.Karthikeyan and G.M.N'guer ekata, Nonlocal Cauchy problem for some fractional abstract integro-differential equations in Banach spaces, Communications Math. Anal., 6 (2009) 31-35.

5. K.Balachandran and F.P.Samuel, Existence of solutions for quasilinear delay integrodifferential equations with nonlocal condition, Electronic J. Diff. Equ., 2009 (2009) 1-7.

6. K.Balachandran and J.J.Trujillo, The nonlocal Cauchy problem for nonlinear fractional integro-differential equations in Banach spaces, Nonlinear Anal. Theory Meth. Applic., 72 (2010) 4587-4593.

7. T.G.Bhaskar, V.Lakshmikantham and S.Leela, Fractional differential equations with Krasnoselskii-Krein-type condition, Nonlinear Anal. Hybrid Sys., 3 (2009) 734-737.

8. D.Delboso and L.Rodino, Existence and uniqueness for a nonlinear fractional differential equation, J. Math. Anal. Appl., 204 (1996) 609-625.

9. K.Diethelm, The Analysis of Fractional Differential Equations, Lecture Notes in Mathematics 2004, Springer, Berlin, 2010.

10. A.A.Kilbas, H.M.Srivastava and J.J.Trujillo, Theory and Applications of Fractional Differential Equations, North-Holland Math. Stud., 204, Elsevier, Amsterdam, 2006.

11. K.S.Miller and B.Ross, An Introduction to the Fractional Calculus and Differential Equations, John Wiley, New York, 1993.

12. S.Momani, A.Jameel and S.Al-Azawi, Local and global uniqueness theorems on fractional integro-differential equations via Bihari's and Gronwall's inequalities, Soochow Journal of Mathematics, 33 (2007) 619.

13. K.Oldham and J.Spanier, The fractional calculus theory and applications of differentiation and integration to arbitrary order, 111, Elsevier, 1974.

14. M.R.Rao, Ordinary Differential Equations, East-West Press PVT Limited, 1980.

15. S.G.Samko, A.A.Kilbas and O.I.Marichev, Fractional Integrals and Derivatives. Theory and Applications, Gordon and Breach, Yverdon, 1993.

16. J.Wu and Y.Liu, Existence and uniqueness of solutions for the fractional integrodifferential equations in Banach spaces. Electronic Journal of Differential Equations, 2009 (2009), 1-8.

17. Y.Zhou, Basic theory of fractional differential equations, 6, Singapore: World Scientific, 2014. 